\title{
A novel approach for APNR \& Vehicle categorization in camera pictures
}

\author{
Satyam Srivastava ${ }^{1}$, Kota Solomon Raju $^{2}$, Shashikant Sadistap $^{3}$ \\ ${ }^{I}$ (M. Tech., AES, Academy of Scientific \& Innovative Research, CSIR-CEERI)) \\ ${ }^{2}$ (Principal Scientist, Digital System Group, CSIR-CEERI) \\ ${ }^{3}$ (Senior Principal Scientist, Agri-Electronics Group, CSIR-CEERI)
}

\begin{abstract}
This paper addresses an improved version of Toll gate system comparative to existing system in market. There are several ANPR systems available in market. In all existing systems the major problems were there limitations as well as limited features. So presented system contains multiple features like one of the major feature is number plate recognition with improved performance, matching with data base etc as well as one other feature also installed in this system, which people already tried with different kind of arrangement and that is vehicle categorization means how system can categories the vehicles based on their weights. People tried this feature with pressure sensors but unfortunately this system didn't work due to less life of the pressure sensors as well as other environment related limitations. This kind of application will very help full in toll gate system applications. Presented system can be categorized in two major blocks first one is ANPR and second one is vehicle categorization based on their weights. This paper presented only simulation part as well as with proposed system. In these systems major sections are plate localization, plate recognition, vehicle categorization. These three are the major parts of this project in which vehicle categorization and plate recognition are the most complex blocks. About data base preparation, initially for trial purpose some vehicle pictures captured from the organization. It is observed that after required improvements system detects $96 \%$ pictures from the prepared data base.
\end{abstract}

Keywords: - APNR, Toll gate, vehicle categorization, plate recognition, plate localization, Hough transform.

\section{INTRODUCTION}

There is a need for intelligent traffic management systems in order to cope with the constantly increasing traffic on today's roads for that we need a intelligent toll gate system which can control large amount of traffic as well as able to maintain the data base regarding vehicles. Generally which we missed in daily life due to human errors. Video based traffic surveillance systems is an important need for such kind of systems. Information about current situations can be automatically extracted by image processing algorithms. Beside vehicle detection and tracking, identification via license plate recognition is important for a variety of applications. These include, e.g. automatic congestion charge systems, access control, tracing of stolen cars, or identification of dangerous drivers. So we maintain the data base for authorized licenses from that we can track unauthorized drivers which is a big issue in any country. Simultaneously if we can able to categorized vehicles based on their weights, so from that we can automatic maintain the toll tax as well as road tax. So in this system not only license plate while as we are also categorizing the vehicles based on their weights like heavy vehicle, light vehicle etc. So we can say that from this system we can incorporate with all existing problems as well as it can be a solution for all existing problems like toll tax management, data base management for allowed vehicles. Automatic License Plate Recognition systems are very popular and studied all over the world. Two main parts of these systems are:

- Finding license plates in images (Plate Localization).

- Reading text from license plates

The problems about the images with license plates are:

- Poor image resolution (the plate is too far away - low quality camera)

- Motion Blur

- Poor lighting and low contrast due to overexposure, reflection or shadows

- Dirt on the plate.

- Life of the pressure sensors. 
Presented system have capability to overcome problems like life of the pressure sensors often used in toll gate systems and improved APNR algorithm have capability to overcome most of the problem related to APNR.

\section{DESCRIPTION OF SYSTEM}

Whole system divided in to two sections first one is APNR and second one is vehicle categorization feature. APNR subsystem divided in to four major blocks which are plate localization, cleaning, segmentation, Statistical and Structural based verification. While as in second subsystem major blocks are tire localization, centers localization, judgment of radius range, circle detection and finally diameter calculation using Hough transform tool. An overall block diagram has been presented as follows.

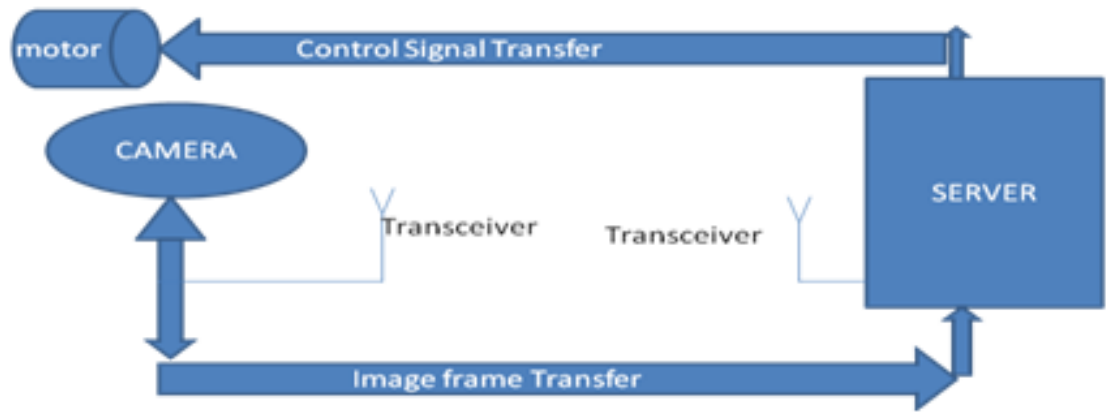

Figure 2.1: Block diagram of overall system

In overall system camera will take frames from two angles first one is front view and second one is side view. After taking frames it will transmit to server where all algorithms already implemented. Initially developed prototype was totally based on embedded but due to less memory processor becomes slow. After transmission of both frames, system extracts all information like number plate, vehicle category and based on category it automatically calculate toll tax. After matching with database if it is authorized number plate then it will transmit a controls signal to other wise it will inform to authority for manual opening. About algorithm part which installed in server are as following.

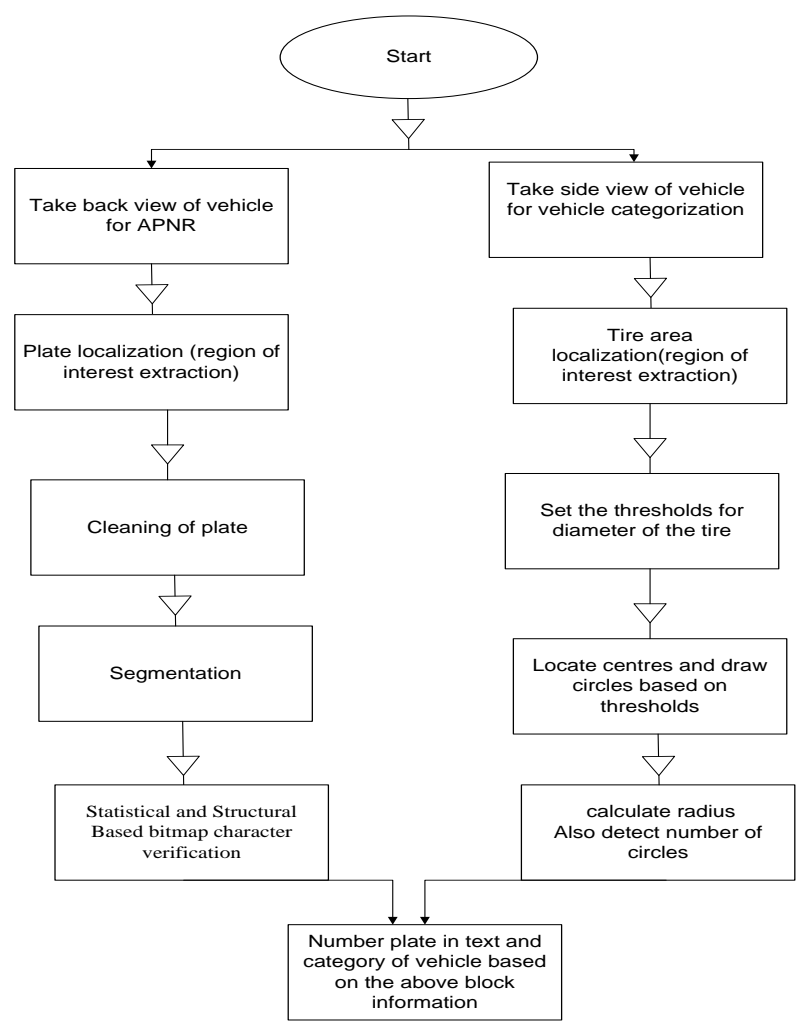

Figure 2.2: Flow diagram of overall system

So as above explained in system flow diagram, system captures two frames from different angle of every vehicle. First one is for number plate and second one is for vehicle categorization. So after frame transfer 
system processed all APNR algorithms on first frame as well as related to tire diameter detection implemented on second frame or side view of the vehicle.

\subsection{Number plate recognition}

Number plate localization block divided in four major blocks presented as follows.

\subsubsection{Localization phase:}

First block in APNR is plate localization in which system extract the region of interest like in case of APNR, region of interest is number plate of the vehicle. In plate localization there are several algorithms exist in literature like basis of edge finding, basis of adaptive thresholding, basis of fuzzy set theory etc. Author tried to implement this block using all three algorithms but finally, based on the performance, author chose basis of edge finding algorithm for system development. It is most successful way for plate localization compare to other methods used for plate localization. It is based on the observation that the license plate is an area in the image with high contrasts, usually composed of black and white or black and yellow characters or objects. The characters or objects on the number plates are organized in one row, or a few rows in India. The largest brightness variation of the rows is taken as the limit of the search and based on the limit of search we find the upper as well as lower position of number plate. The edge finding algorithm is applied to the whole image as a first step for the purpose of highlighting the high contrast areas that are characteristic of number plates. Then we checked based on this range in both row profile and column profile and the maximum intensity amplitude is the starting point of the plate. Similarly we do for row profile and after this process we located the number plate, which is the region of interest for plate localization block.

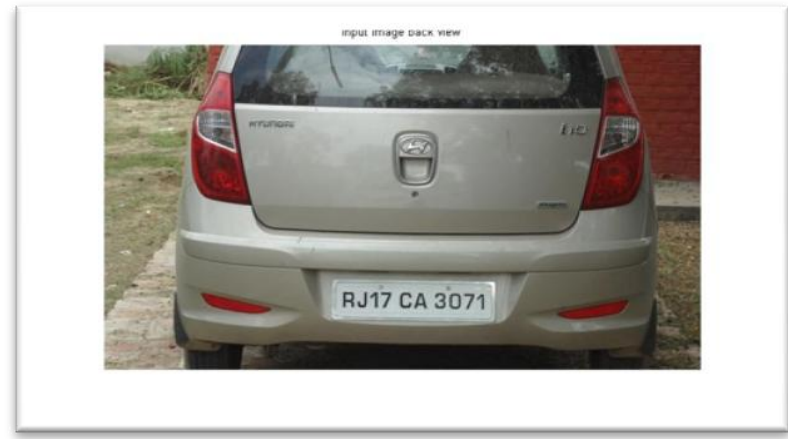

a)

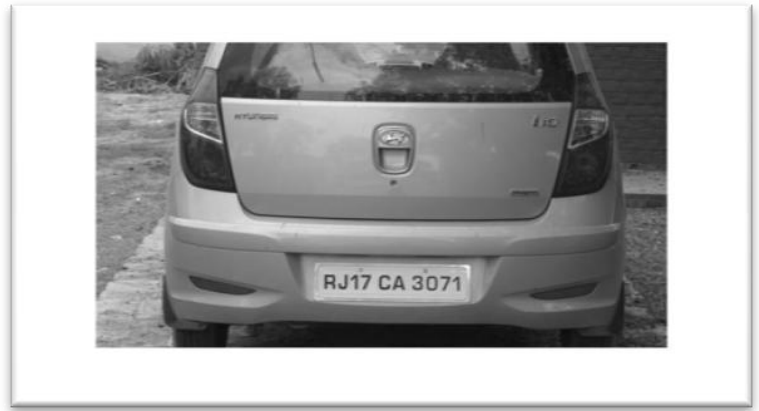

b)

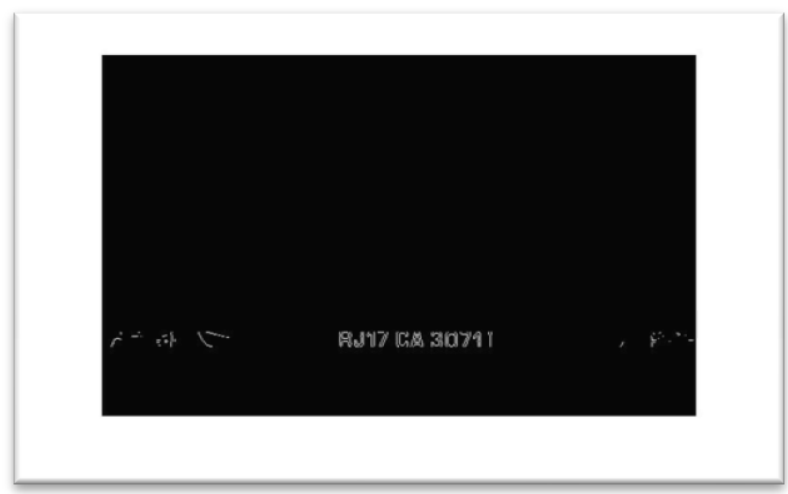

c) 


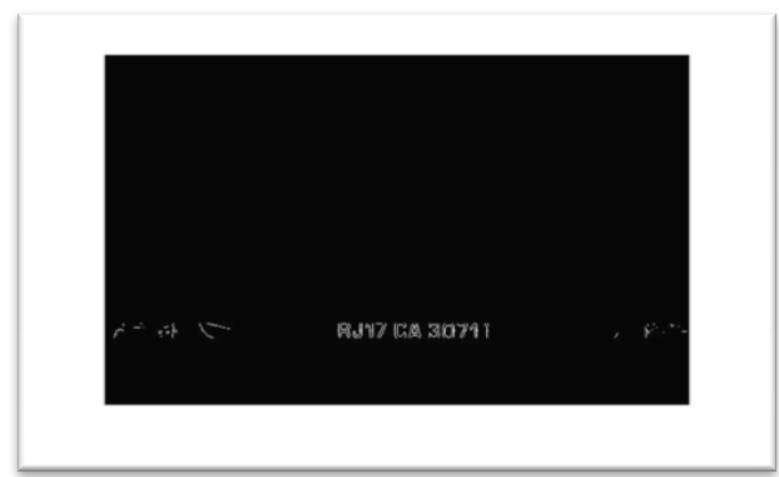

d)

Figure 2.1.1.1: a) Source image from CSIR-CEERI campus b) Grayscale conversion c) Number plate located d) After hard thresholding

\subsubsection{Plate enhancement phase (cleaning phase):}

This block increases the interpretability of number plate using cleaning phase. In this phase basically system removes black spots which are unnecessary information for our system. Hard thresholding, convolution, and rank order filters have been used for cleaning as well as smoothing purpose. First system set a threshold based on image and based on that threshold system binaries whole image and remove unnecessary information from number plate. After that rank order filters have been used for smoothing purpose .These filters will reduce the noise as well as blur part comes in the frame. In this system selects a $3 \mathrm{X} 3$ mask and move it on whole number plate image. After smoothed image has been generated, system generates a white strip have same size comparative to number plate and convolute both. After this whole process, this block generated a cleaned version of number plate generated from previous block.

\begin{tabular}{|l|l|l|}
\hline 1 & 1 & 1 \\
\hline 1 & 1 & 1 \\
\hline 1 & 1 & 1 \\
\hline
\end{tabular}

a)

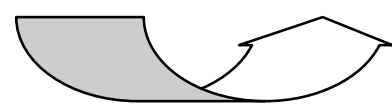

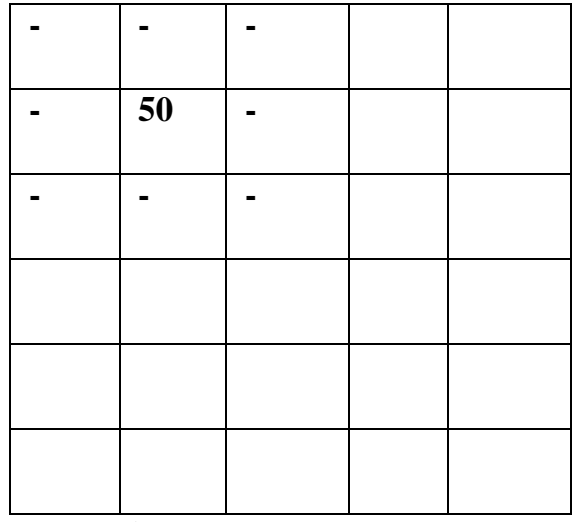

b) c)

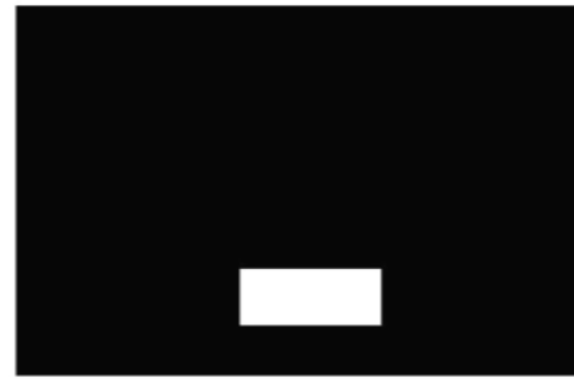

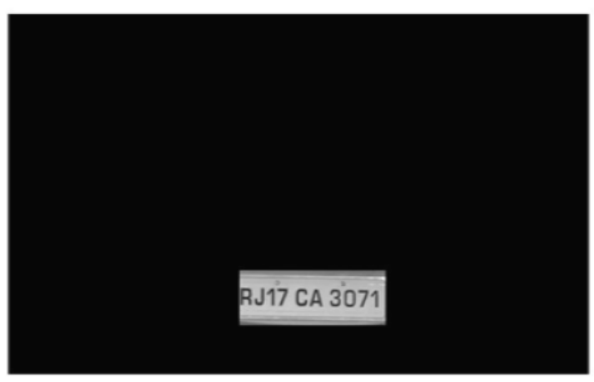

d)

Figure 2.1.2.1: a) Mask of averaging filter b) Mask of number plate c) Black number plate generated for convolution d) Final image of number plate localization 


\subsubsection{Segmentation phase:}

This block performs segmentation on cleaned version of the number plate. In this phase system segments different characters from cleaned version of number plate. Traditional techniques have been used like histogram segmentation for segmentation purpose. In this technique, system segments all characters based on generated histogram overshoots and undershoots (Figure 2.1.3.1 b) of the number plate. Number plate is a collection of objects have different black and white strips. Whenever system detects an object in number pate, a group of overshoots also detected on the same time. After every single object a histogram undershoots comes in the number plate histogram. System performs this process iteratively until all characters have been segmented.

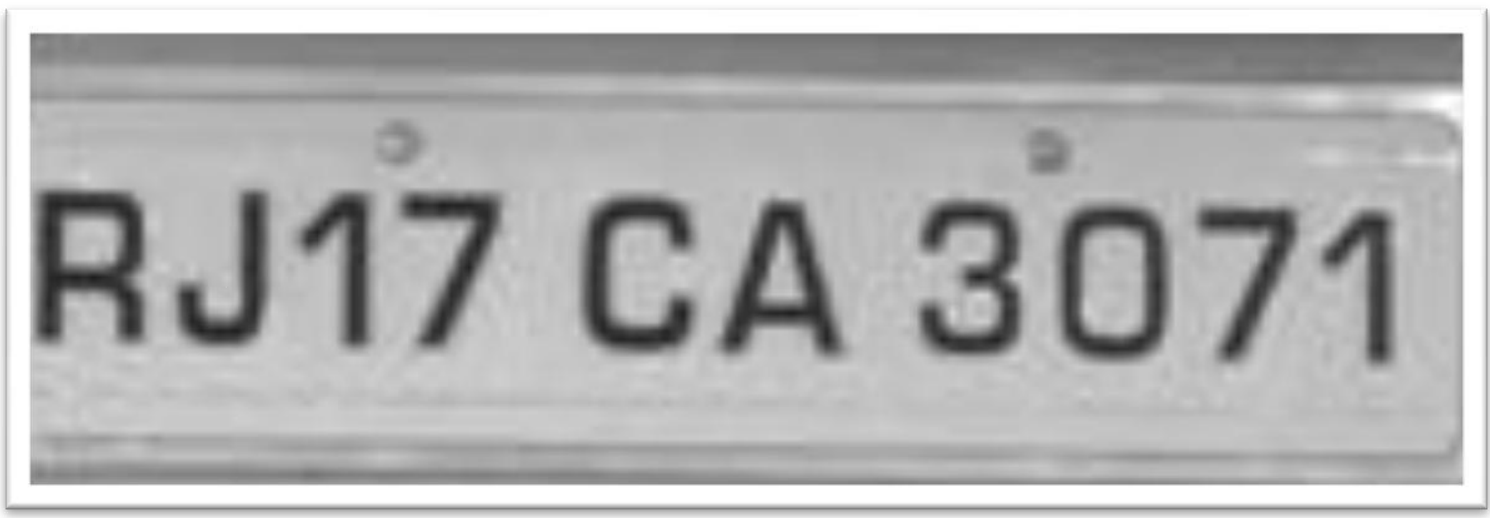

a)

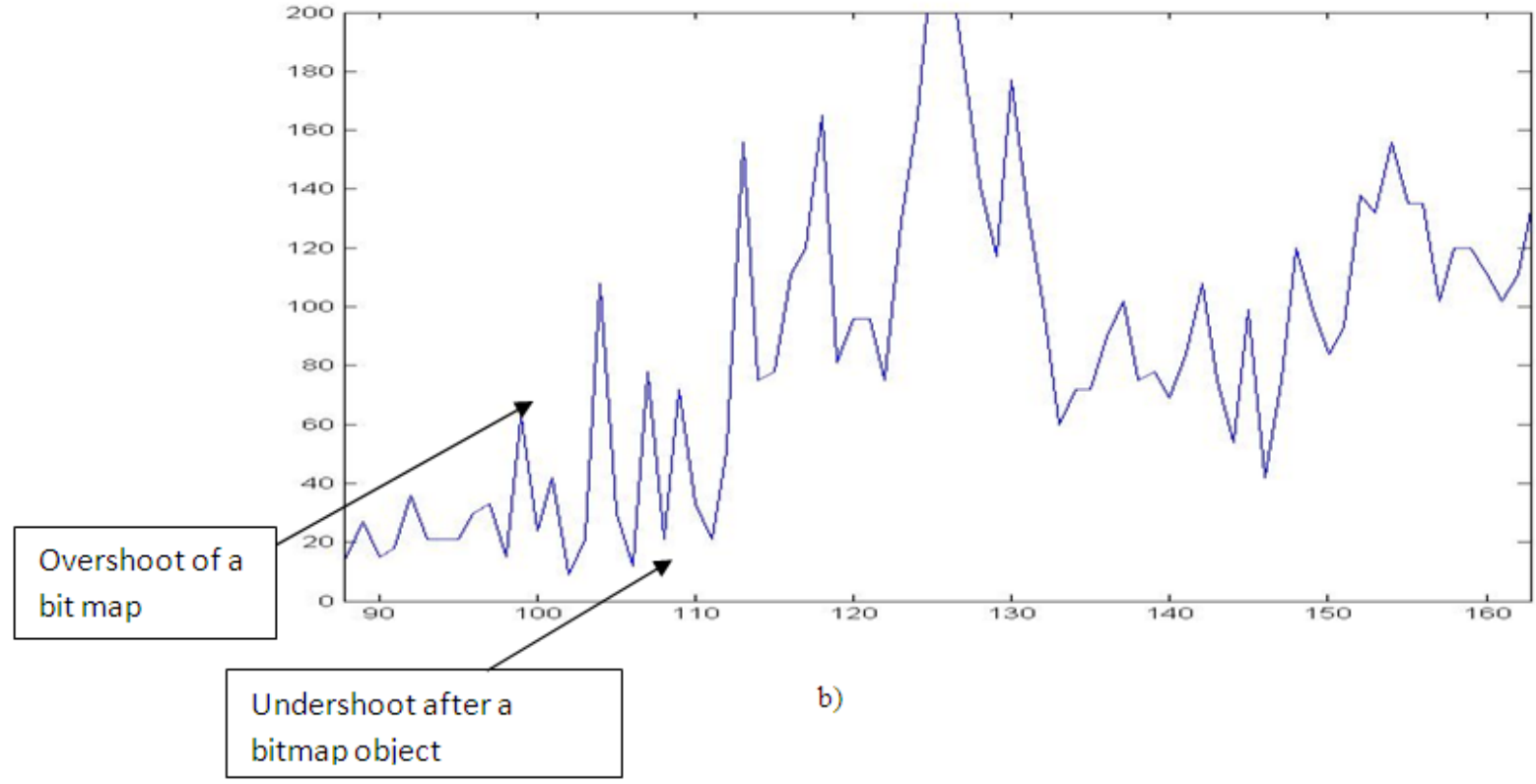

Figure 2.1.3.1: a) Number Plate b) Histogram based segmentation

\subsubsection{Statistical and Structural verification:}

This block performs statistical structural verification on the segmented number plate image generated from previous block. People used this technique in daily life for recognizing the different patterns like as shown in figure 2.1.4.1 a) triangle can be recognized based on its statistical information like number of segments, number of diagonals, number of vertices etc. Similarly this technique applied on segmented bitmap number plate image like character ' $\mathrm{A}$ ' has 3 joints, 2 end points, 5 limbs etc. It is also possible that two different characters contain similar statistical information. So, in that case next preferred technique in series is structural verification. This is also same kind of technique but contains different kind of information like as shown in figure 2.1.4.1 a) triangle can be recognized using its structural information. Similarly this technique applied on segmented number plate image like character 'A' contains a triangle with two tilted limbs. 


\section{Statistical}

Number of segments

Number of horizontal segments: 2

Number of /vertical segments : 2

Number of diagonal segments : 0

Number of segments $: 3$

Number of horizontal segments : 1

Number of /vertical segments : 0

Number of diagonal segments : 2

\section{Structural}
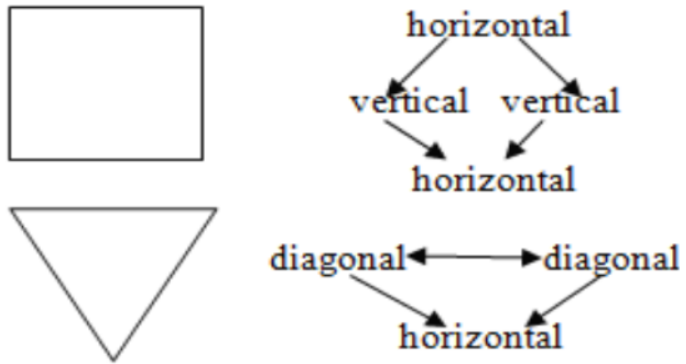

a)

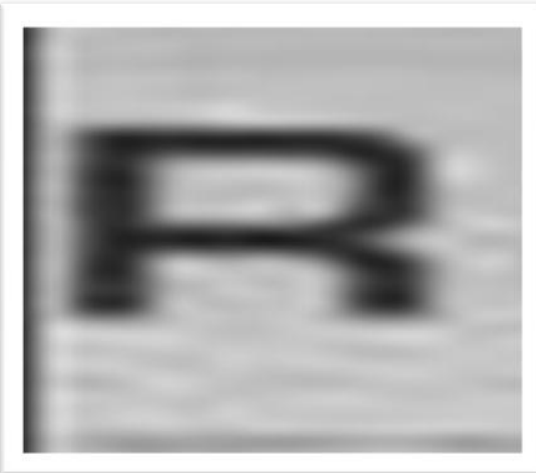

b)

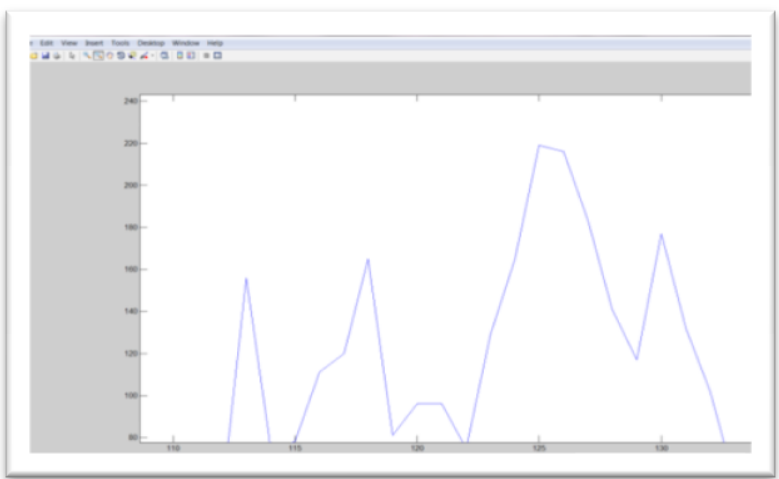

c)

Figure 2.1.4.1: a) Basic statistical and structural approach b) Bitmap character of number plate c) Column profile of character have 4 peaks

\subsection{Vehicle categorization}

Vehicle categorization block have been divided in to two major subsections presented as follows.

\subsubsection{ROI extraction:}

This section addresses the region of interest extraction from the capture frames as presented in figure 2.2.1.1 a). In this process region of interest is the location of tire of the vehicles. System captures two side frames as well as one front frame from installed camera from different angles. Front frames uses for number plate recognition as well as other two frames used for vehicle categorization process. System locates tire portion based on fact that tire portion exists lower part of the frame. So basically system divides whole side frame in to two frames and lower frame as shown in figure 2.2.1.1 c) contains the region of interest portion. After that system extracts single tire from the lower part of the frame based on the fact that tire is a combination of two annular disks have black and white rings. After extraction process has been completed some basic preprocessing process also performed on the final extracted image as shown in figure 2.2.1.1 b) like averaging, smoothing, noise removal etc.

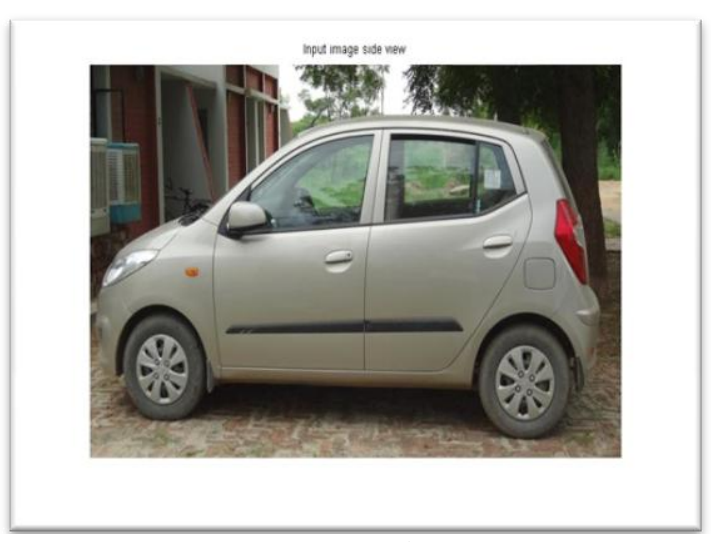

a)

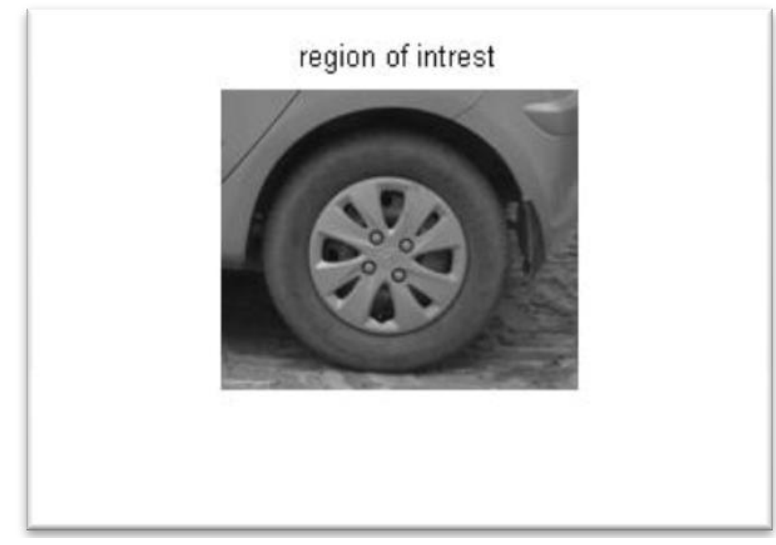

b) 


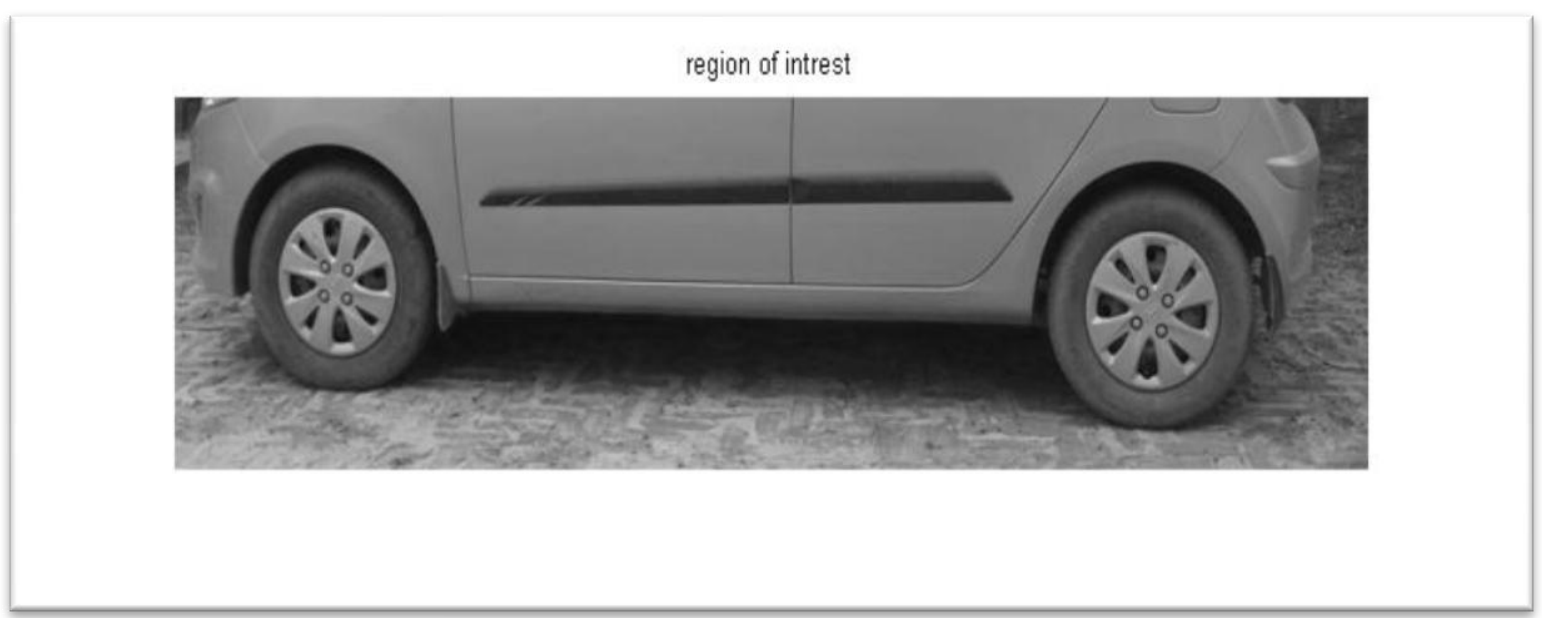

c)

Figure 2.2.1.1: a) Side view of vehicle b) First region of interest c) Second region of interest

\subsubsection{Diameter and number of tires calculation:}

All other blocks have been merged in this section as explained in above flow diagram of the system. This section focused on the diameter extraction of the tire and number of tires calculation. Traditional Hough transform have been used for this process. There is a common problem in machines for recognizing the number of orientation of a particular object in a given image. Often objects in a picture are the combination of different mathematical shapes like line, circle, ellipse etc. Hough transform is a technique which can be used to isolate features of a particular shape within an image. There are so many applications of Hough transform but here we discuss about circles as per as our application. So about circle detection we know the basic parametric equation of circle as following.

$$
(x-a)^{2}+(y-b)^{2}=r^{2}
$$

where $x, y$ are the locus points of cicles and $(a, b)$ is center of the circle and $r$ is the radius of the circle.

$$
\begin{aligned}
& x=a+r * \cos \theta \\
& y=b+r * \sin \theta
\end{aligned}
$$

where where $x, y$ are the locus points of cicles and $(a, b)$ is center of the circle, $r$ is the radius of the circle and $\theta$ is parametric cordinate of the circle.

Traditional hough transform tool has been used for tire detection as well as radius detection with require improvement. Firrst step is turn colored image in to grayscale after that system creates a 3D hough array (accumlator) with the first two dimensions representing the cordinates of the circle origin and third dimensions represents the radius. After creation of 3D hough array, in the next step system detect the outer edges of the circle as well as simultaneously system increments the corresponding elements in the hough array. After all above mentioned steps system draw circles around tire segment .

The curve obtained in the Hough Transform space for each edge point will be a right circular cone in three dimension and intersection of cones will give the informtion about parameters $(a, b, r)$. Then system performs edge detection on source image after that on each edge points we draw a circle of expected radius. After that intersection of all circles is the original center of the circle. Simultaneously after every center detection system increases accumlator by one. Accumlator is nothing just a kind of counter, which provides the infpormation about number of circles detection.

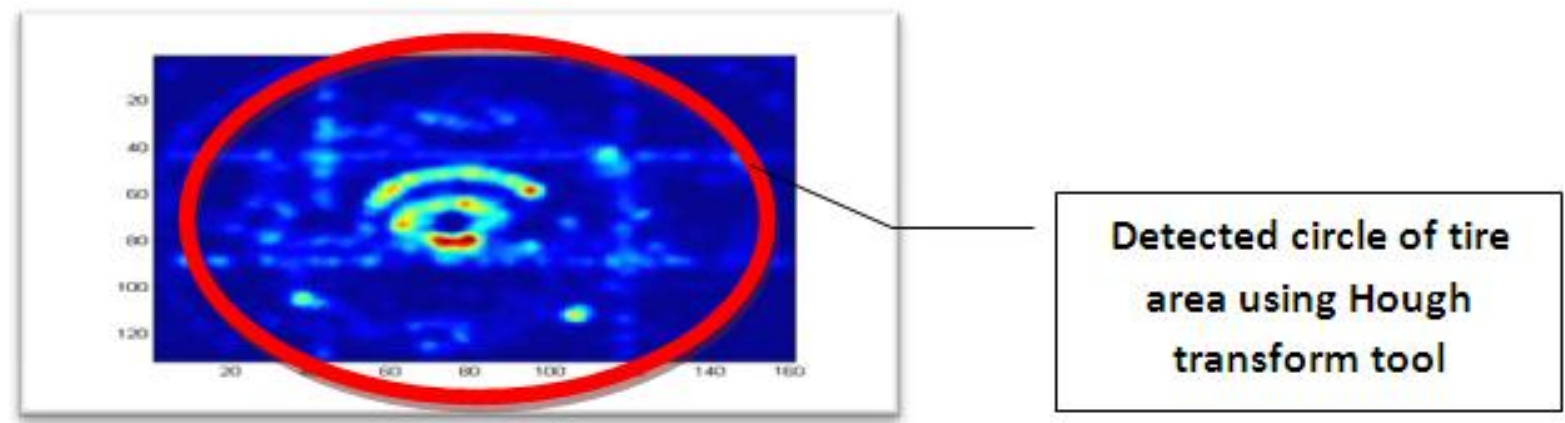

a) 


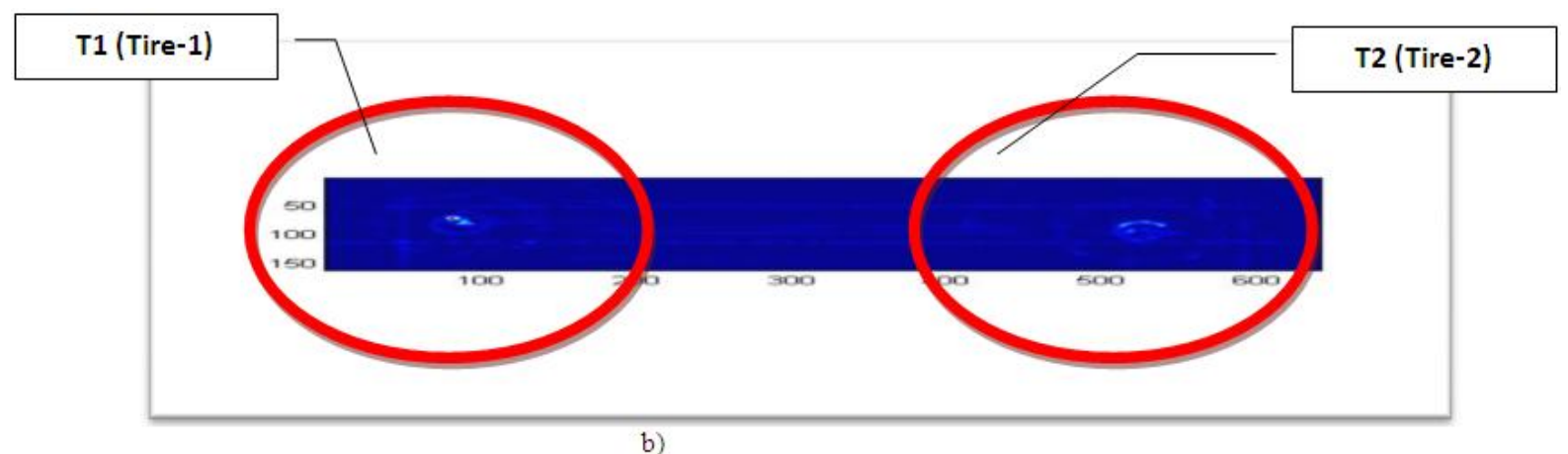

Figure 2.2.2.1: a) circle detection for diameter extraction b) circle detection for number of circles calculation

Tire segment detected using hough transform tool as well as simultaneously detected approximate radius of detected circle but there is a need of training of the system for match with approximate radius of the detected circle using hough transform tool and measured radius of the different vechiles.

\section{RESULTS AND DISCUSSIONS}

Raw Image with Circles Detected (center positions and radii marked)

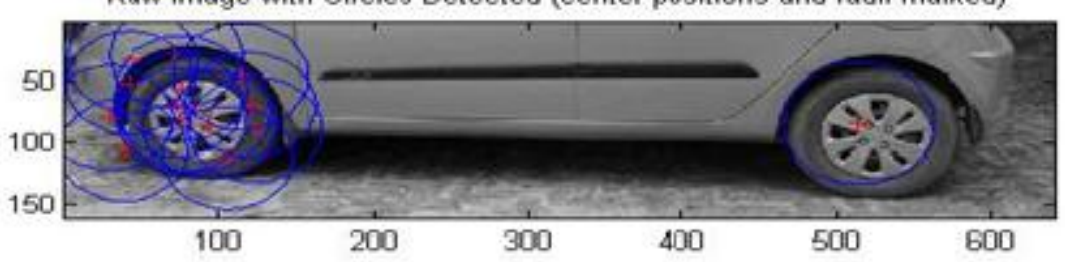

a)

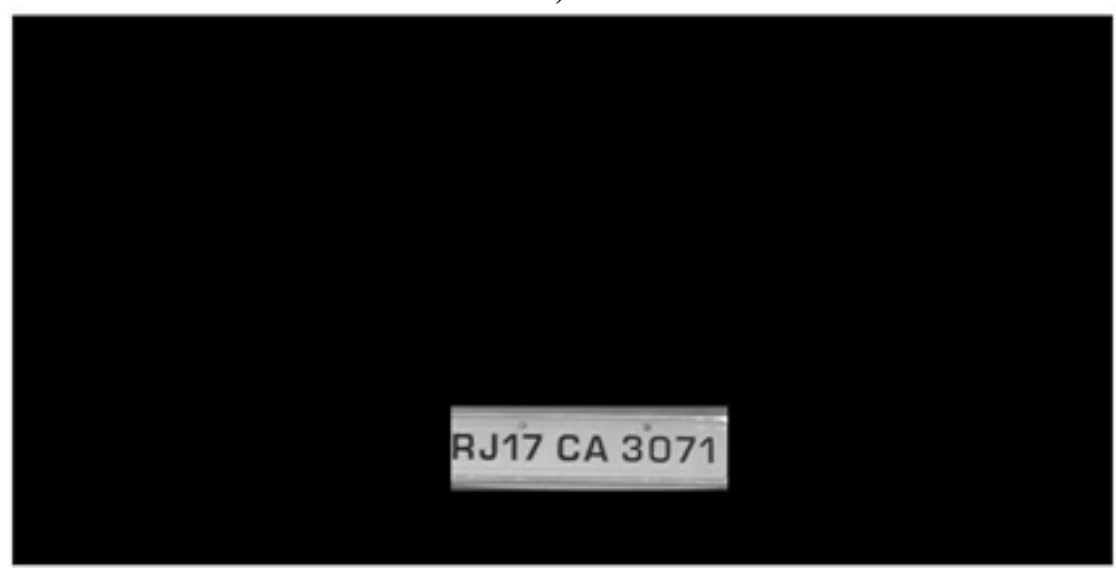

b)

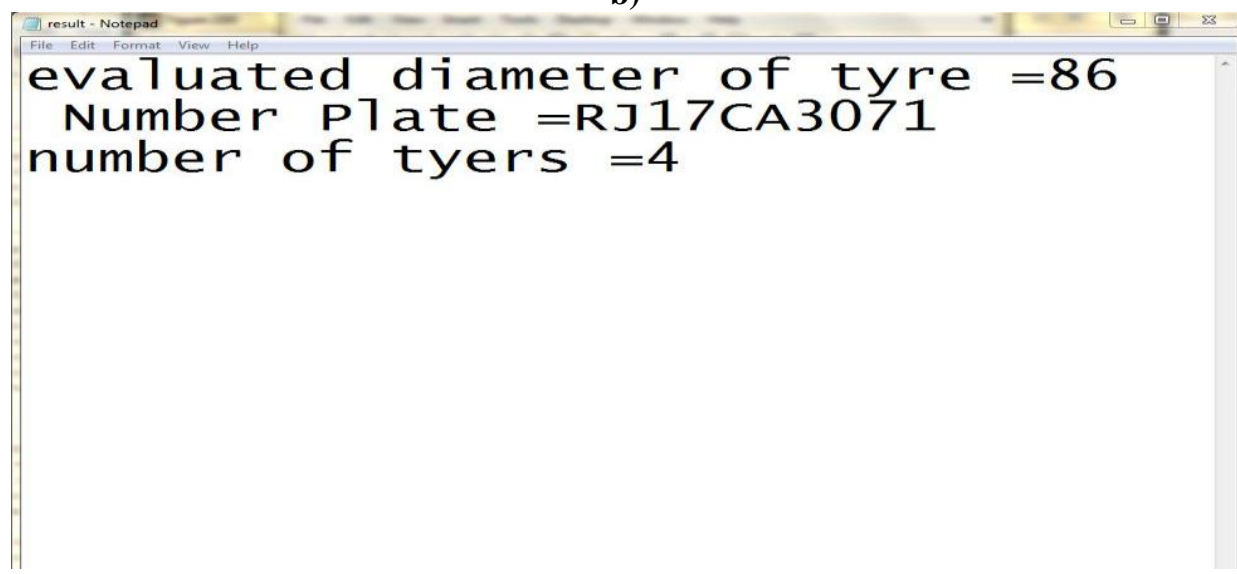

c)

Figure 3.1: a) circle detection for diameter extraction b) Located number plate c) overall results 


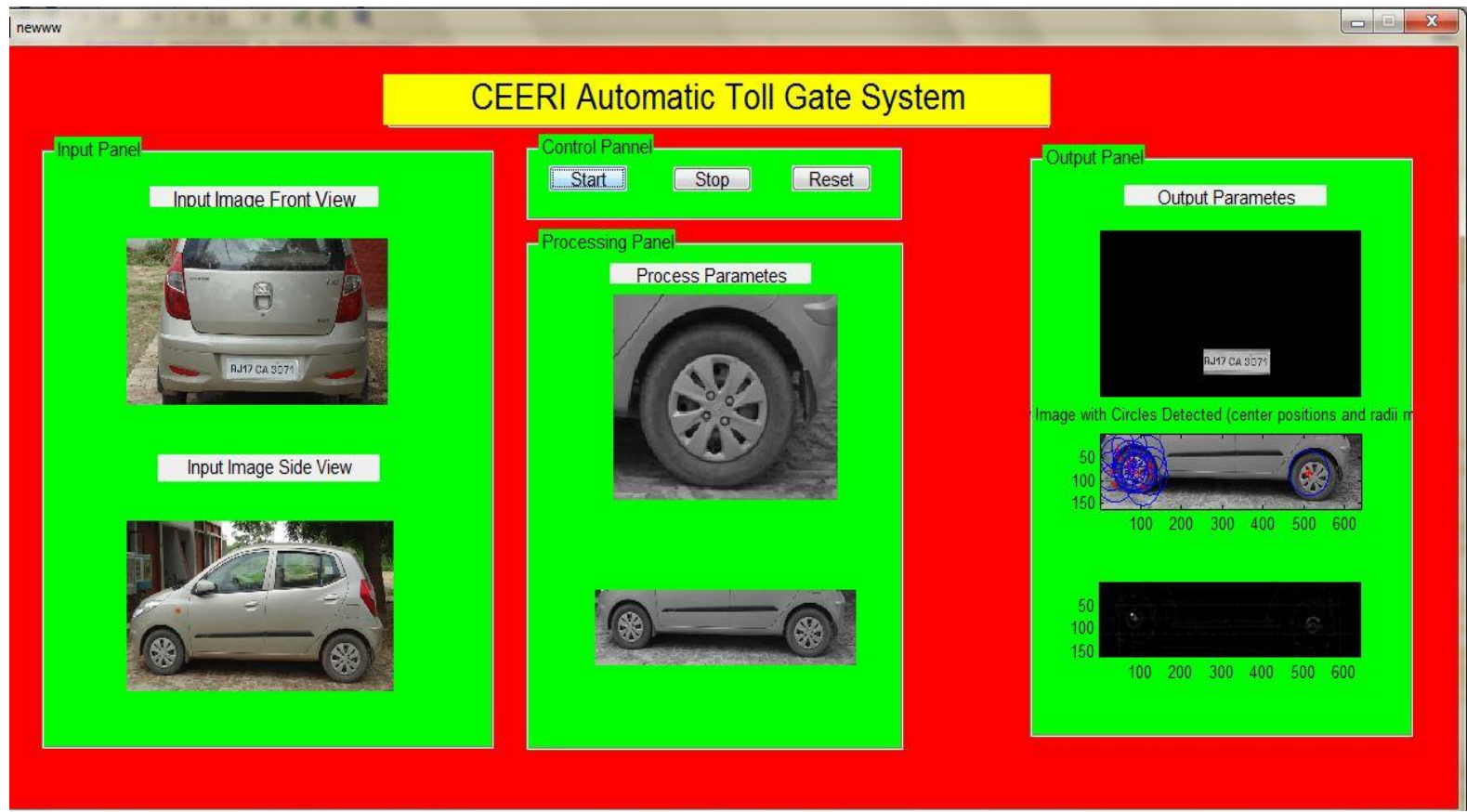

Figure 3.2: GUI of the overall system

Figure 3.1a) presents detection of the tire for vechile categorization and b) section presents finall located number plate after all the processing performed like claening, localization etc while as in c) section presents the overall results saved in text format contains the information about number plate, approximate diameter and number of tires.

Table 3.1 Comparison of proposed algorithm with other algorithms based on live testing

\begin{tabular}{|l|l|l|l|l|l|}
\hline Algorithms & $\begin{array}{l}\text { Total } \\
\text { samples } \\
\text { (images) }\end{array}$ & $\begin{array}{l}\text { Correct } \\
\text { recognition }\end{array}$ & False recognition & Efficiency (\%) & $\begin{array}{l}\text { Simulation } \\
\text { time(sec.) }\end{array}$ \\
Parameter
\end{tabular}

So as per as performance analysis and achieved results are better comparative to other algorithms, since the existing ALPS had the most problems in classification phase. Therefore, it was not surprising that these phases performed better than the segmentation and cleaning phase. The overall result was improved because as it can easily figure out from above table that efficiency of the proposed algorithms is much better comparative other algorithms, even not only efficiency, simulation time of proposed algorithm is better or we can say that proposed algorithm is fast comparative to other algorithms.

\section{CONCLUSION AND FUTURE ASPECT}

The location and classification phase are most complex blocks and draw more attention comparative to other blocks, since the existing APNR had the most problems there. Therefore, it was not surprising that these phases performed better than the segmentation and cleaning phase. A new feature, vehicle categorization is also implemented and adds with the whole APNR system. The overall result was satisfy able and improved comparative to previous existing versions. 
There is still a scope of improvement of vehicle categorization feature algorithm. Traditional Hough transform needs to improve based on its detection performance and also there is a need of to develop overall system on embedded platform for decreasing the cost as well as size of the system

\section{ACKNOWLEDGEMENT}

Author is grateful to Director CSIR-CEERI for providing support to carry out project work at Central Electronics Engineering Research Institute (CSIR-CEERI). Author is also grateful to CEERI members for allowing us to use their vehicle images for project work.

[1] R.A. Lotufo, A.D. Morgan, and AS. Johnson, 1990, "Automatic Number-Plate Recognition," Proceedings of the IEEE Colloquium On Image analysis for Transport Applications, V01.035, pp.6/16/6,February 16, 1990.

[2] Xiaojun Zhai, Faycal Bensaali and RezaSotudeh, 2012 “OCR-Based Neural Network for ANPR”.

[3] Maged M. M. FAHMY, 1994 “Automatic Number Plate Reconition using neural network”.

[4] Prathamesh Kulkarni (Student Member, IEEE), Ashish Khatri, Prateek Banga, Kushal Shah, 2009, Automatic Number Plate Recognition (ANPR) System for Indian conditions

[5] C. Arth, F. Limberger, H. Bischof, "Real Time Plate Recognition on an Embedded DSP Platform", in Proc. IEEE Conf. CVPR, Jun., 2007, pp. 1-8

[6] G. Cao, J. Chen, and J. Jiang, "An Adaptive Approach to Vehicle License Plate Localization", 2003 Proceedings of the IEEE Colloquium On Image analysis for Transport Applications.

[7] Ketelaars, Nicole. "Final Project: Automated License Plate Recognition." AIMe Magazine 1 (2002): 9-12.

[8] Ballard, Dana H. "Generalizing the Hough transform to detect arbitrary shapes."Pattern recognition 13.2 (1981): 111-122.

[9] Xu, Lei, Erkki Oja, and Pekka Kultanen. "A new curve detection method: randomized Hough transform (RHT)." Pattern recognition letters 11.5 (1990): 331-338.

[10] Mahmoud Ibrahim, Mohamed Shehata,2012, “Automatic License PlateRecognition (ALPR): A State-ofthe-Art Review".

[11] Vesna Zeljković, Pavel Praks, Robert Vincelette, Claude Tameze, Ladislav Válek, 2009, “Automatic Pattern Classification of Real Metallographic Images".

[12] Ping Dong, Jie-hui Yang, Jun-jun Dong, 2006, "The Application and Development Perspective of Number Plate Automatic Recognition Technique" Proceedings of the ICTTA Colloquium On Digital Object Identifier. 